\title{
QUASICOINCIDENCE FOR INTUITIONISTIC FUZZY POINTS
}

\author{
FRANCISCO GALLEGO LUPIÁÑEZ
}

Received 22 September 2004 and in revised form 29 March 2005

The introduction of intuitionistic fuzzy sets is due to K. T. Atanassov, who also proposed some problems about this subject. D. Çoker defined the intuitionistic fuzzy topological spaces and, with some coworkers, studied these spaces. In this paper, we define and study the notion of quasicoincidence for intuitionistic fuzzy points and obtain a characterization of continuity for maps between intuitionistic fuzzy topological spaces

The introduction of "intuitionistic fuzzy sets" is due to Atanassov [1], and this theory has been developed in many papers $[2,3,4]$. This author proposed as an open problem "to investigate the topological and geometric properties of the IFSs" remarking that "some first steps in this direction are made" [4].

In this paper, we define for intuitionistic fuzzy sets the notion of quasicoincidence and the corresponding neighborhood structure (see [9]). These concepts allow us to obtain a characterization of continuity for maps between two intuitionistic fuzzy topological spaces.

(For notions on ordinary fuzzy topology used in this paper, see $[7,8]$.)

First, we list some previous definitions.

Definition 1 [1]. Let $X$ be a nonempty set. An intuitionistic fuzzy set (IFS) $A$ of $X$ is an object having the form

$$
A=\left\{\left\langle x, \mu_{A}, \gamma_{A}\right\rangle \mid x \in X\right\}
$$

where the functions $\mu_{A}: X \rightarrow I$ and $\gamma_{A}: X \rightarrow I$ denote the degree of membership and the degree of nonmembership of each element $x \in X$ to an ordinary subset of $X$, and $0 \leq \mu_{A}(x)+\gamma_{A}(x) \leq 1$ for each $x \in X$.

Notation 2. $0_{\sim}=\langle x, 0,1\rangle$ and $1_{\sim}=\langle x, 1,0\rangle$.

Definition 3 [2]. Let $X$ be a nonempty set, and let $A$ and $B$ be two IFSs of $X$. Then,

(a) $A \subseteq B$ if $\mu_{A}(x) \leq \mu_{B}(x)$ and $\gamma_{A}(x) \geq \gamma_{B}(x)$;

(b) $A=B$ if $A \subseteq B$ and $B \subseteq A$; 
1540 Quasicoincidence for intuitionistic fuzzy points

(c) $A \cup B=\left\{\left\langle x, \mu_{A} \vee \mu_{B}, \gamma_{A} \wedge \gamma_{B}\right\rangle \mid x \in X\right\}$;

(d) $A \cap B=\left\{\left\langle x, \mu_{A} \wedge \mu_{B}, \gamma_{A} \vee \gamma_{B}\right\rangle \mid x \in X\right\}$.

Definition 4 [5]. Let $\left\{A_{j} \mid j \in J\right\}$ be an arbitrary family of IFSs of $X$. Then,

(a) $\cap A_{j}=\left\{\left\langle x, \wedge \mu_{A j}, \vee \gamma_{A j}\right\rangle \mid x \in X\right\}$;

(b) $\bigcup A_{j}=\left\{\left\langle x, \vee \mu_{A j}, \wedge \gamma_{A j}\right\rangle \mid x \in X\right\}$.

(For other definitions concerning IFSs used in this paper, see $[5,6]$.)

Definition 5. Let $A=\left\{\left\langle x, \mu_{A}, \gamma_{A}\right\rangle \mid x \in X\right\}$ and $B=\left\{\left\langle x, \mu_{B}, \gamma_{B}\right\rangle \mid x \in X\right\}$ be two IFSs. Say that $A$ quasicoincides with $B$, denoted by $A q B$ if $\mu_{A}$ quasicoincides with $\mu_{B}$ and $\gamma_{A}^{\prime}$ quasicoincides with $\gamma_{B}^{\prime}$.

Remark 6. If $A q B$, we have that $A \cap B \neq 0_{\sim}$. $\left(\mu_{A} q \mu_{B}\right.$ implies that $\mu_{A} \wedge \mu_{B} \neq 0$, then $A \cap B \neq 0 \sim)$.

Remark 7. If $A$ and $B$ verify that $\gamma_{A}=\mu_{A}^{\prime}$ and $\gamma_{B}=\mu_{B}^{\prime}$, then $A q B$ if, and only if, $\mu_{A} q \mu_{B}$.

Proposition 8. Let $A$ and $B$ be two IFSs of $X$, let $f: X \rightarrow Y$ be a map between two nonempty sets $X$ and $Y$, then if $A q B, f(A) q f(B)$.

Proof. $A q B$ if and only if $\mu_{A} q \mu_{B}$ and $\gamma_{A}^{\prime} q \gamma_{B}^{\prime}$. Then, we have that $f\left(\mu_{A}\right) q f\left(\mu_{B}\right)$ and $f\left(\gamma_{A}^{\prime}\right) q f\left(\gamma_{B}^{\prime}\right)$, that is, $\left(1-f\left(\gamma_{A}^{\prime}\right)\right)^{\prime} q\left(1-f\left(\gamma_{B}^{\prime}\right)\right)^{\prime}$. Thus $f(A) q f(B)$.

Proposition 9. Let $X$ and $Y$ be two nonempty sets, let $f: X \rightarrow Y$ be a map, let $A$ be an IFS of $X$, and let $C$ be an IFS of $Y$. If $f(A) q C, A q f^{-1}(C)$.

Proof. $f(A) q C$ if and only if $f\left(\mu_{A}\right) q \mu_{C}$ and $f\left(\gamma_{A}^{\prime}\right) q \gamma_{C}^{\prime}$. Then, $\mu_{A} q f^{-1}\left(\mu_{C}\right)$ and $\gamma_{A}^{\prime} q f^{-1}\left(\gamma_{C}^{\prime}\right)$, that is, $\gamma_{A}^{\prime} q\left(f^{-1}\left(\gamma_{C}\right)\right)^{\prime}$ (because $\left.f^{-1}\left(\gamma_{C}^{\prime}\right)=f^{-1}\left(\gamma_{C}\right)^{\prime}\right)$.

Remark 10. If $A, B$, and $C$ are IFSs of $X$, such that $A q B$, and $B \subseteq C$, then $A q C$.

Definition 11. Let $(X, \tau)$ be an IFTS, and let $p$ be an IFP of $X$. Say that an IFS $N$ of $X$ is a $Q$-neighborhood of $p$ if there exists an $\operatorname{IFOS} A$ of $(X, \tau)$ such that $p q A$ and $A \subseteq N$.

Theorem 12. Let $(X, \tau)$ be an IFTS, let $p$ be an IFP of $X$, and let $u_{Q}(p)$ be the family of all the $Q$-neighborhoods of $p$ in $(X, \tau)$, then,

(1) $N \in U_{Q}(p)$ implies that $p q N$,

(2) $N_{1}, N_{2} \in u_{Q}(p)$ imply that $N_{1} \cap N_{2} \in u_{Q}(p)$,

(3) if $N \in \mathcal{U}_{Q}(p)$ and $N \subseteq M$, then $M \in \mathcal{U}_{Q}(p)$,

(4) if $N \in \mathcal{U}_{Q}(p)$, there exists $M \in \mathcal{U}_{Q}(p), M \subseteq N$, such that, for every IFP e which quasicoincides with $M, M \in \mathcal{U}_{Q}(e)$.

Proof. (1) $N \in U_{Q}(p)$ if and only if there exists an IFOS $A$ such that $p q A$ and $A \subseteq N$, then $p q N$ (by Remark 10).

(2) $N_{1}, N_{2}$ are $Q$-neighborhoods of $p$ if and only if there exist two IFOSs $A_{i}$ such that $p q A_{i}, A_{i} \subseteq N_{i}(i=1,2)$, then, if $p=c(\alpha, \beta)$, we have that $c_{\alpha} q \mu_{A_{i}}, \mu_{A_{i}} \leq \mu_{N_{i}}, c_{1-\beta} q \gamma_{A_{i}}^{\prime}, \gamma_{A_{i}} \geq$ $\gamma_{N_{i}}(i=1,2)$, then $c_{\alpha} q \mu_{A_{1} \cap A_{2}}, \mu_{A_{1} \cap A_{2}} \leq \mu_{N_{1} \cap N_{2}}, c_{1-\beta} q \gamma_{A_{1} \cap A_{2}}^{\prime}, \gamma_{A_{1} \cap A_{2}} \geq \gamma_{N_{1} \cap N_{2}}$, and $p q\left(A_{1} \cap\right.$ $\left.A_{2}\right), A_{1} \cap A_{2} \subseteq N_{1} \cap N_{2}$, with $A_{1} \cap A_{2}$ an IFOS.

(3) It is obvious. 
(4) $N \in U_{Q}(p)$ if and only if there exists an IFOS $A$ such that $p q A$ and $A \subseteq N$, then $A$ is also a $Q$-neighborhood of $p$, and for each IFP $e$ such that $e q A, A$ is a $Q$-neighborhood of $e$.

Proposition 13. Let $X$ be a nonempty set, for each IFP $p$ of $X$, let $\boldsymbol{u}_{Q}(p)$ be a family of IFSs verifying (1), (2), and (3) of the theorem, then $\tau=\left\{U\right.$ IFS $\mid U \in \mathcal{U}_{Q}(p)$ if $\left.p q U\right\}$ is an IFT in X. If also the family verifies (4), then $u_{Q}(p)$ is the system of $Q$-neighborhoods of $p$ in $(X, \tau)$.

Proof. $1_{\sim} \in \tau$ by $(3)$.

$U_{i} \in \tau(i=1,2)$, and $p q\left(U_{1} \cap U_{2}\right)$, then $U_{i} \in \mathcal{U}_{Q}(p)$ and $U_{1} \cap U_{2} \in \mathcal{U}_{Q}(p)$ by (2).

$\left\{U_{j}\right\}_{j \in J} \in \tau, p q \cup U_{j}$ with $p=c(\alpha, \beta)$ if and only if $c_{\alpha} q \mu_{\cup U_{j}}$ and $c_{1-\beta} q \gamma_{\cup U_{j}}^{\prime}$ and it is equivalent to $c_{\alpha} q \mu \cup U_{j_{0}}$ (for some $j_{0}$ of $J$ ) and $c_{1-\beta} q \gamma_{\cup U_{j}}^{\prime}$ (for all $j$ of $J$ ). Then $p q U_{j_{0}}$ for some $j_{0} \in J, U_{j_{0}} \in \mathcal{U}_{Q}(p)$ for some $j_{0} \in J$, and $\cup U_{j} \in U_{Q}(p)$ by (3).

Finally, if $N \in U_{Q}(p)$, there exists $M \in \mathcal{U}_{Q}(p), M \subseteq N$, such that for every IFP $e$ which quasicoincides with $M$, we have that $M \in \mathcal{U}_{Q}(e)$, then $M \in \tau, p q M, M \subseteq N$, and $N$ is a $Q$-neighborhood of $p$ in $(X, \tau)$. Conversely, for every $Q$-neighborhood $N$ of $p$ in $(X, \tau)$, there is an $A \in \tau$ such that $p q A, A \subseteq N$, then for every IFP $e$ which quasicoincides with $A$, we have that $A \in \mathcal{U}_{Q}(e)$, thus $N \in \mathcal{U}_{Q}(p)$.

Proposition 14. Let $X, Y$ be two nonempty sets, let $f: X \rightarrow Y$ be a map, let $\tau$ be an IFT in $X$, and let $s$ be an IFT in $Y$. Then, $f:(X, \tau) \rightarrow(Y, s)$ is continuous if, and only if, for each IFP $p$ of $X$, and for each Q-neighborhood $V$ of $f(p)$, there exists a $Q$-neighborhood $U$ of $p$ such that $f(U) \subseteq V$.

Proof. If $V$ is a $Q$-neighborhood of $f(p)$, there exists an IFOS $G$ such that $f(p) q G$ and $G \subseteq V$, then $p q f^{-1}(G)$ (by Proposition 9), and $f^{-1}(G)$ is an IFOS such that $f^{-1}(G) \subseteq$ $f^{-1}(V)$. Thus, $f^{-1}(V)$ is a $Q$-neighborhood of $p$ and $f\left(f^{-1}(V)\right) \subseteq V$.

Conversely, for each $G \in s$, we have that, for every IFP $p$ such that $p q f^{-1}(G)$ is $f(p) q f\left(f^{-1}(G)\right)$ (by Proposition 8), then $f(p) q G$, and $G$ is a $Q$-neighborhood of $f(p)$. By the hypothesis, there exists a $Q$-neighborhood $U$ of $p$ such that $f(U) \subseteq G$, then $U \subseteq$ $f^{-1}(G)$ and $f^{-1}(G) \in U_{Q}(p)$. From Proposition 13, it follows that $f^{-1}(G) \in \tau$.

\section{References}

[1] K. T. Atanassov, Intuitionistic fuzzy sets, VII ITKR's Session, Sofia, 1983 (Central Sci. Tech. Library, Bulg. Acad. Sci, 1984).

[2] _ Intuitionistic fuzzy sets, Fuzzy Sets and Systems 20 (1986), no. 1, 87-96.

[3] More on intuitionistic fuzzy sets, Fuzzy Sets and Systems 33 (1989), no. 1, 37-45.

[4] Intuitionistic Fuzzy Sets. Theory and Applications, Studies in Fuzziness and Soft Computing, vol. 35, Physica-Verlag, Heidelberg, 1999.

[5] D. Çoker, An introduction to intuitionistic fuzzy topological spaces, Fuzzy Sets and Systems 88 (1997), no. 1, 81-89.

[6] D. Çoker and M. Demirci, On intuitionistic fuzzy points, Notes IFS 1 (1995), no. 2, 79-84.

[7] Y.-M. Liu and M.-K. Luo, Fuzzy Topology, Advances in Fuzzy Systems-Applications and Theory, vol. 9, World Scientific, New Jersey, 1997.

[8] N. Palaniappan, Fuzzy Topology, CRC Press, Florida, 2002. 


\section{Quasicoincidence for intuitionistic fuzzy points}

[9] P. M. Pu and Y.-M. Liu, Fuzzy topology. I. Neighborhood structure of a fuzzy point and MooreSmith convergence, J. Math. Anal. Appl. 76 (1980), no. 2, 571-599.

Francisco Gallego Lupiáñez: Departamento de Geometría y Topología, Facultad de Ciencias Matemáticas, Universidad Complutense de Madrid, 28040 Madrid, Spain

E-mail address: fg_lupianez@mat.ucm.es 


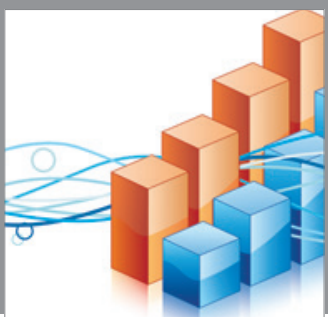

Advances in

Operations Research

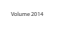

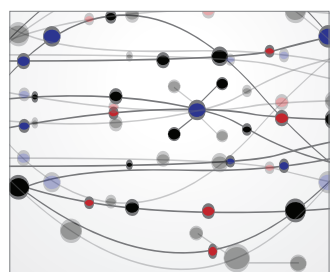

\section{The Scientific} World Journal
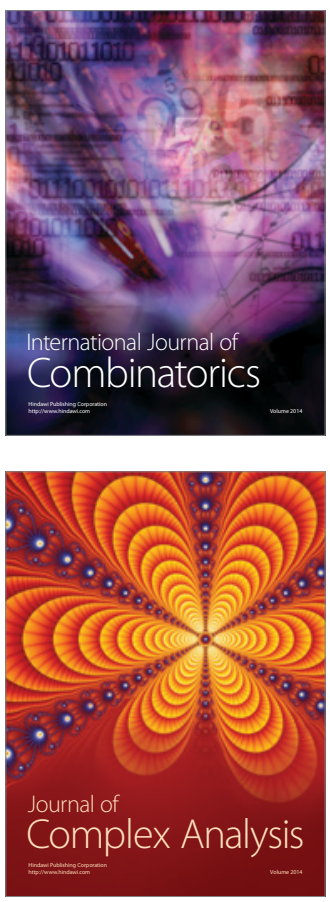

International Journal of

Mathematics and

Mathematical

Sciences
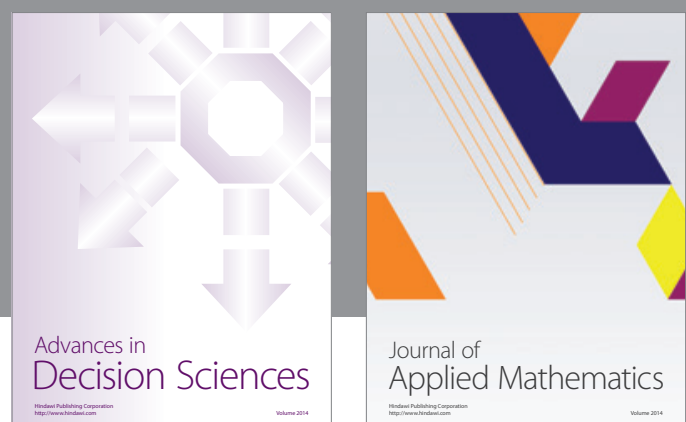

Journal of

Applied Mathematics
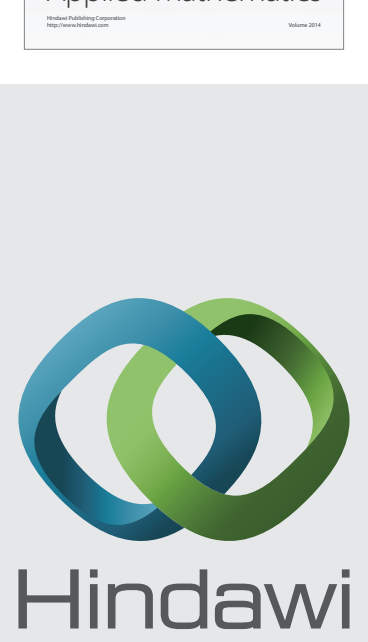

Submit your manuscripts at http://www.hindawi.com
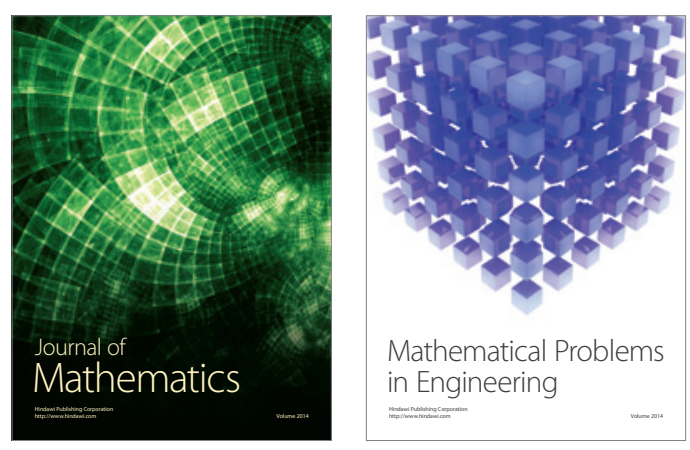

Mathematical Problems in Engineering
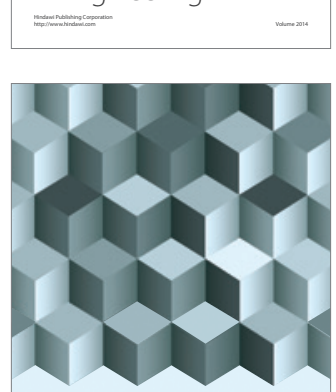

Journal of

Function Spaces
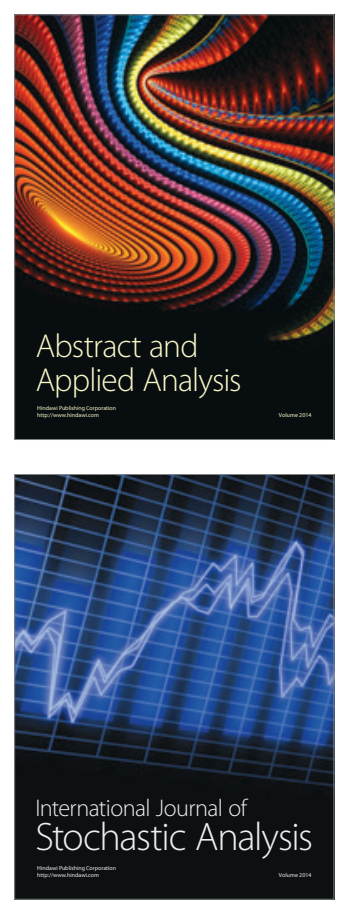

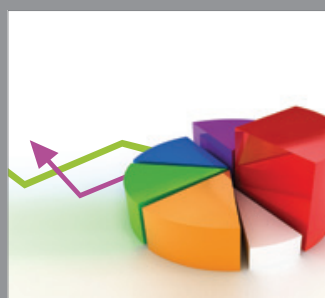

ournal of

Probability and Statistics

Promensencen
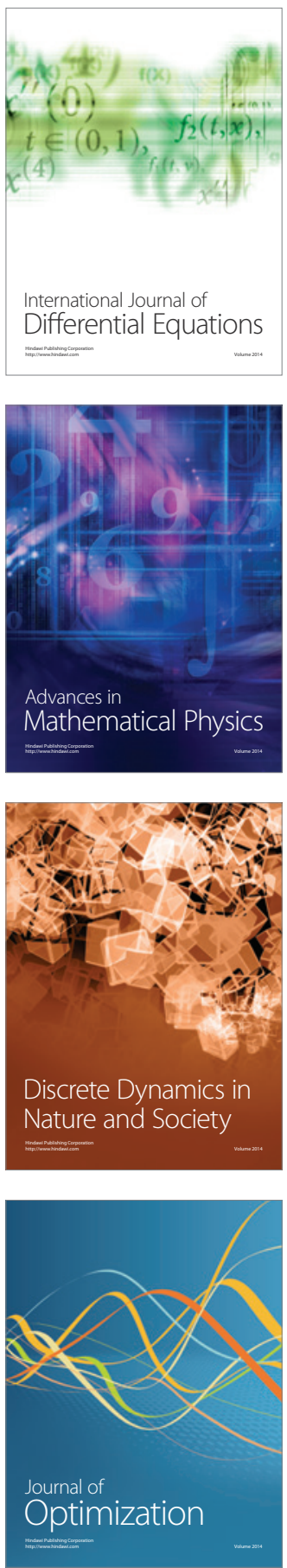